Gut and Liver, Vol. 13, No. 1, January 2019, pp. 32-39

\title{
Assessment of Clinical Outcomes after Peroral Endoscopic Myotomy via Esophageal Distensibility Measurements with the Endoluminal Functional Lumen Imaging Probe
}

\author{
In Kyung Yoo ${ }^{1}$, Sang Ah $\mathrm{Choi}^{1}$, Won Hee Kim${ }^{1}$, Sung Pyo Hong ${ }^{1}$, Ozlem Ozer Cakir ${ }^{2}$, and Joo Young $\mathrm{Cho}^{1}$ \\ ${ }^{1}$ Department of Gastroenterology, CHA Bundang Medical Center, CHA University School of Medicine, Seongnam, Korea, and ${ }^{2}$ Department of \\ Gastroenterology, Alanya Alaaddin Keykubat University, School of Medicine, Antalya, Turkey
}

See editorial on page 3.

Background/Aims: Endoluminal functional lumen imaging probe (EndoFLIP) is a modality that enables clinicians to measure volume-controlled distension of the esophagus. This study aimed to assess the utility of EndoFLIP in patients who had achalasia treated with peroral endoscopic myotomy (POEM). We hypothesized that improvement in the distensibility index (DI) is correlated with the postoperative clinical outcome of POEM. Methods: Patients who underwent POEM for achalasia at Cha Bundang Medical Center were included. Physiological measurements of the lower esophageal sphincter (LES) pressure before and after POEM were assessed using EndoFLIP. Patients' symptoms were recorded using the Eckardt score. Results: A total of 52 patients with achalasia were included in this study. Patients with a post-POEM DI below 7 (30 or $40 \mathrm{~mL}$ ) had a significantly higher rate of incomplete response after POEM ( $p=0.001)$. Changes in LES pressure or integrated relaxation pressure after POEM were also significantly associated with an incomplete response $(p=0.026$ and $p=0.016$, respectively). Multivariate analysis showed that post-POEM DI $<7$ was the most important predictor of an incomplete response after POEM ( $p=0.004)$. Conclusions: Lower post-POEM DI values were associated with an incomplete post-POEM response. Therefore, postPOEM DI at the esophagogastric junction using EndoFLIP is a useful index for predicting the clinical outcome of POEM in patients with achalasia. (Gut Liver 2019;13:32-39)
Key Words: Esophageal achalasia; Peroral endoscopic myotomy; Functional lumen imaging probe; Treatment outcome

\section{INTRODUCTION}

Achalasia is a rare chronic motility disorder of the esophagus with symptoms of dysphagia, regurgitation, aspiration, and chest pain; it has an incidence of 1 per 100,000 per year. ${ }^{1}$ The pathophysiology of achalasia is related to selective degeneration of inhibitory neurons in the myenteric plexus of the esophagus. This affects the smooth muscle layer of the lower esophageal sphincter (LES), resulting in impaired relaxation and loss of coordinated peristalsis of the esophagus. ${ }^{2}$ Achalasia is usually diagnosed using a combination of modalities including esophagogastroduodenoscopy, gastrografin esophagogram, esophageal high-resolution manometry (HRM), and computed tomography (CT). The traditional treatments for achalasia are pneumatic balloon dilatation, botulinum toxin injection, and surgical treatment with Heller's myotomy. ${ }^{3}$ Pneumatic balloon dilation and Heller's myotomy improve physiologic parameters of the lower esophagus, such as the distensibility index (DI), LES pressure, and esophageal emptying of the esophagogastric junction (EGJ). ${ }^{4}$ Although botulinum toxin injection has been shown to have excellent short-term results, it has limited utility due to the short duration of symptomatic relief, recurrence of symptoms, and complications of submucosal fibrosis. Even though Heller's myotomy is an invasive treatment for achalasia and provides the maximum symptom relief, it has the drawbacks of long duration of hospitalization, high cost, pain, and wound infection. Peroral endoscopic myotomy (POEM) is a novel endoscopic

Correspondence to: Joo Young Cho

Department of Gastroenterology, CHA Bundang Medical Center, CHA University School of Medicine, 59 Yatap-ro, Bundang-gu, Seongnam 13496, Korea

Tel: +82-31-780-5641, Fax: +82-31-780-5005, E-mail: cjy6695@dreamwiz.com

Received on May 22, 2018. Revised on July 7, 2018. Accepted on July 8, 2018. Published online December 14, 2018

pISSN 1976-2283 eISSN 2005-1212 https://doi.org/10.5009/gnl18233

In Kyung Yoo and Sang Ah Choi contributed equally to this work as first authors.

@ This is an Open Access article distributed under the terms of the Creative Commons Attribution Non-Commercial License (http://creativecommons.org/licenses/by-nc/4.0) which permits unrestricted non-commercial use, distribution, and reproduction in any medium, provided the original work is properly cited. 
treatment for achalasia that was developed by Inoue et $a l .^{5}$ in 2008. Thereafter, POEM has been used as a minimally invasive alternative; it is reported to have excellent clinical outcomes. ${ }^{6}$ However, there is a lack of objective tools to determine the extent of myotomy and impairment of relaxation after POEM. Although a decrease in LES pressure to less than $10 \mathrm{~mm} \mathrm{Hg}$ has been reported as a predictor of favorable long-term clinical outcomes after pneumatic balloon dilatation, ${ }^{7,8}$ few studies have assessed the predictive role of such parameters after POEM. Endoluminal functional lumen imaging probe (EndoFLIP) is a modality to complement conventional diagnostic tools like gastrografin esophagogram and HRM. EndoFLIP evaluates the physiologic measurements of the esophagus during volumecontrolled distension. It uses impedance planimetry for real-time measurement of the cross-sectional area (CSA) and pressure of the EGJ. DI is defined as the CSA divided by the pressure and represents the degree of impaired LES relaxation and increased LES pressure. The aim of this study was to assess the long-term efficacy of POEM and define the usefulness of EndoFLIP in evaluating the clinical response to POEM. We hypothesized that the improvement in the parameters measured using EndoFLIP, such as DI and CSA, correlates to the postoperative clinical outcome of POEM.

\section{MATERIALS AND METHODS}

\section{Patients}

A single-institute retrospective study was conducted to assess the utility of EndoFLIP. Patients who underwent POEM at the gastroenterology center of CHA university Bundang Hospital between November 2014 and November 2017 were included in this study. Physiologic measurements of LES before and after POEM were assessed with esophagogastroduodenoscopy, HRM, 24-hour pH monitoring, gastrografin esophagogram, and EndoFLIP. Chest CT was performed to exclude extrinsic compression or structural deformity of the esophagus. Symptoms related to achalasia were assessed according to the Eckardt score and recorded. The gastroenterologic examinations above were performed based on the outpatient clinic department or during hospitalization. If the examinations were performed after hospitalization, chest CT, esophagogastroduodenoscopy, EndoFLIP, HRM, and 24-hour pH monitoring were performed on the first post-admission day and gastrografin esophagogram was performed on the 2nd day. Patients were allowed a soft diet for 4 days and returned to a normal diet on the 5th day after POEM. Intravenous anesthetics, parenteral nutrition, and standard doses of proton pump inhibitors were administered during the fasting period. Informed consent was obtained from all subjects. The procedures were conducted in accordance with the ethical standards of our Hospital Ethics Committee and Institutional Review Board. This study was approved by the Institutional Review Board of CHA Bundang Medical Center (registration number:

\section{CHAMC 2018-07-050-001).}

\section{POEM technique}

With the patients under general anesthesia, POEM was performed by two skilled endoscopists (J.Y.C and I.K.Y) following the techniques described by Inoue et al. ${ }^{5}$ Submucosal injection was administered at 9 to $10 \mathrm{~cm}$ above the EGJ. After an incision was made at the lower esophageal mucosa, submucosal tunnel was created on the basis of endoscopic submucosal dissection technique using dual knife and hook knife. Longitudinal myotomy of the inner circular muscle layer of the esophagus was made along the lower esophagus and was extended through the LES until about 2 to $3 \mathrm{~cm}$ into the gastric cardia. The myotomy length was measured with an endoscope (GIF-HQ290; Olympus, Tokyo, Japan) during POEM. Full-thickness myotomy was performed if the patients had severe symptoms of achalasia or were diagnosed with type III achalasia. Closure of the incision site of the submucosal tunnel was performed using hemoclips.

\section{EndoFLIP}

EndoFLIP (Crospon Ltd., Galway, Ireland) was recommended at least twice for all patients treated with POEM. EndoFLIP was performed before and after POEM using a single-use EndoFLIP catheter (EF-325N) by well-trained endoscopists at an outpatient clinic department. Antispasmodic drugs were discontinued at least 7 days prior to EndoFLIP. Esophagogastroduodenoscopy was done just before EndoFLIP to measure esophageal length. The probe and pressure transducers of EndoFLIP were precalibrated before measurement. The DI of EGJ was measured at the point of narrowest circumference of the functional lumen image during volumetric distension of the saline bag and was expressed in $\mathrm{mm}^{2} / \mathrm{mm} \mathrm{Hg}$. After the probe was inserted throughout the pre-assessed length of the esophagus using an endoscope, the DI and CSA of the EGJ were measured at the site of the narrowest planimetric point. CSA and DI were measured twice during volumetric distension of the saline bag, each of them with $30 \mathrm{~mL}$ (CSA30mL, DI30mL) and $40 \mathrm{~mL}$ (CSA40mL, DI40mL). EndoFLIP was performed 1 month after POEM.

\section{Symptom score}

Symptoms associated with achalasia were assessed with the Eckardt score. Eckardt score contains patient information regarding dysphagia, regurgitation, retrosternal pain, and weight loss. Patients were requested to answer the questionnaires for assessing Eckardt score. Eckardt score was measured more than twice during hospitalization, each of them before and after POEM. After discharge, Eckardt score was checked regularly at the outpatient clinic department during the follow-up period. The final Eckardt score was defined as the lastly recorded Eckardt score. Incomplete clinical response was defined as final Eckardt score $\geq 3$. 


\section{Endoscopy}

Patients underwent esophagogastroduodenoscopy (GIF-Q260; Olympus) one day before POEM to rule out mechanical obstruction and evaluate the grade of reflux esophagitis. Endoscopy was performed 4 days after POEM to observe the recovery state of the esophageal mucosa and to assess delayed adverse events. Follow-up endoscopy was performed in outpatient clinic department after discharge to evaluate the development of reflux esophagitis. Reflux esophagitis was classified according to the Los Angeles classification. ${ }^{9}$

\section{High-resolution manometry}

Physiologic parameters of esophagus including LES pressure and integrated relaxation pressure (IRP) were measured using HRM (Insight G3 HRiM, Sandhill) for differential diagnosis of esophageal motility disorders. Patients with achalasia were classified into subtypes according to LES, IRP, and features of pressurization on EGJ.

\section{Twenty-four-hour pH monitoring}

The 24-hour $\mathrm{pH}$ monitoring was performed using gastroesophageal $\mathrm{pH}$ meter (zepHr, Sandhill). Monitoring was performed after discontinuation of a proton pump inhibitor or $\mathrm{H} 2$ antagonist for at least 7 days. The probe was inserted transnasally into the lower esophagus and the sensor was located approximately 3 to $5 \mathrm{~cm}$ proximal to the EGJ. Measurement was performed continuously for 24 hours. Abnormal acid exposure was defined as percentage of total reflux time (esophageal $\mathrm{pH}$ $<4)$ above 5\%.

\section{Timed gastrografin esophagogram}

Real-time videos and spot images at 1 to 5 minutes after ingestion of $50 \mathrm{~mL}$ of gastrografin contrast agent were obtained. The features of the lower esophagus, as assessed using gastrografin esophagogram, were classified as sigmoid or non-sigmoid type. Follow-up study was performed 4 days after POEM to confirm if leakage occurred at the EGJ.

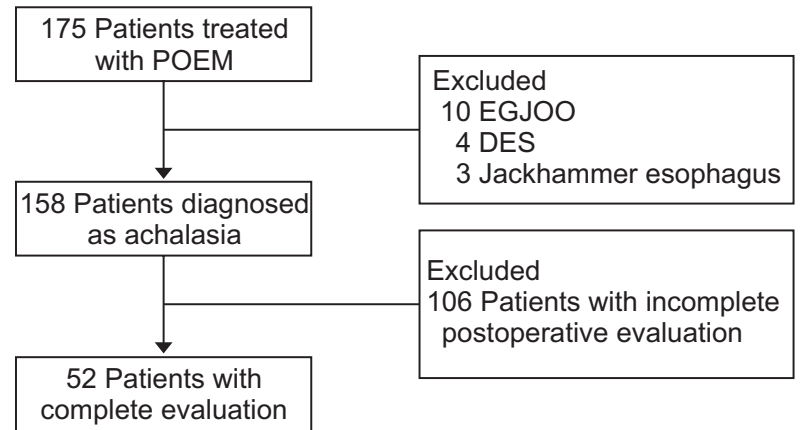

Fig. 1. Patient enrollment.

POEM, peroral endoscopic myotomy; EGJ00, esophagogastric junction outflow obstruction; DES, distal esophageal spasm.

\section{Data analysis and statistical analysis}

Data were presented as mean \pm standard deviation. Statistical analysis was performed using SPSS 24.0 (IBM Corp., Armonk, NY, USA). Chi-square test was performed to identify the correlation between parameters of EndoFLIP and postoperative clinical outcome of POEM. Changes in Eckardt score after POEM were analyzed using paired t-test. The cutoff value of post-DI associated to incomplete clinical response and developing GERD after POEM was determined using receiver operating characteristic (ROC) analysis. Multivariate analysis with logistic regression test was performed to identify factors associated with incomplete clinical response. A two-tailed p-value of $<0.05$ was regarded as statistically significant.

\section{RESULTS}

\section{Patients}

A total of 175 patients (79 males, 96 females; mean age, $43.72 \pm 15.58$ years) underwent POEM for various esophageal

Table 1. Baseline Characteristics $(n=52)$

\begin{tabular}{|c|c|}
\hline Variable & Value \\
\hline \multicolumn{2}{|l|}{ Sex } \\
\hline Male & 27 (51.9) \\
\hline Female & $25(48.1)$ \\
\hline Age, yr & $42.5 \pm 15.2$ \\
\hline BMI, $\mathrm{kg} / \mathrm{m}^{2}$ & $23.2 \pm 3.7$ \\
\hline Follow-up duration after POEM, day & $288.9 \pm 255.3$ \\
\hline \multicolumn{2}{|l|}{ Prior treatment } \\
\hline None & $30(57.7)$ \\
\hline Calcium channel blocker & $4(7.7)$ \\
\hline Balloon dilatation & $4(7.7)$ \\
\hline Botulinum toxin injection & $12(23.1)$ \\
\hline POEM & $2(3.8)$ \\
\hline \multicolumn{2}{|l|}{ Esophageal shape } \\
\hline Sigmoid type & $4(7.7)$ \\
\hline Non-sigmoid type & $48(92.3)$ \\
\hline Maximal diameter of esophagus, $\mathrm{mm}$ & $4.3 \pm 6.3$ \\
\hline Myotomy length, cm & $9.1 \pm 1.8$ \\
\hline \multicolumn{2}{|l|}{ Myotomy method } \\
\hline Selective myotomy & $14(26.9)$ \\
\hline Full myotomy & $38(73.1)$ \\
\hline Procedure time, $\min$ & $73.8 \pm 33.1$ \\
\hline \multicolumn{2}{|l|}{ Type of achalasia } \\
\hline I & $21(40.4)$ \\
\hline II & $26(50)$ \\
\hline III & $5(9.6)$ \\
\hline
\end{tabular}

Data are presented as number $(\%)$ or mean \pm SD. BMI, body mass index; POEM, peroral endoscopic myotomy. 
motility disorders. There were 158 patients with achalasia, 10 with EGJ outflow obstruction (EGJO0), three with jackhammer esophagus, and four with distal esophageal spasm. Among 158 patients diagnosed with achalasia, 106 were excluded due to lack of data or follow-up loss; finally, 52 patients were enrolled (Fig. 1). The baseline characteristics of 52 achalasia patients are shown in Table 1 . The mean age was $42.5 \pm 15.2$ years and 27 patients were male (51.9\%). About $73 \%$ of patients underwent full-thickness myotomy and the rest underwent selective circular myotomy. Among the 52 patients, four (7.7\%) showed sigmoid-like tortuous dilatation of the lower esophagus on a timed gastrografin esophagogram. Four and 14 patients had a history of treatment with balloon dilatation and botulinum toxin injection on the EGJ, respectively. Two patients had a history of POEM treatment and underwent re-operation due to recurrence of symptoms. Four patients (7\%) were diagnosed with type III achalasia, the most complicated subtype.

Table 2 shows the physiologic characteristics of the lower esophagus and EGJ. The IRP and LES pressure measured with HRM decreased significantly after POEM. Increase in DI30mL, DI40mL, CSA30mL, and CSA40mL was also noted. There was a significant decrease in Eckardt score in all patients (Fig. 2). The EndoFLIP tool provides real-time information on the distensibility of the EGJ after POEM and therefore can provide visual effectiveness and predict the outcome of the patient (Fig. 3). The number of patients who were diagnosed with reflux esophagi- tis and gastroesophageal reflux disease (GERD) increased after POEM; 24 (46\%) patients showed endoscopic findings consistent with reflux esophagitis and 15 (28\%) patients aggravated GERD on 24-hour $\mathrm{pH}$ monitoring after POEM.

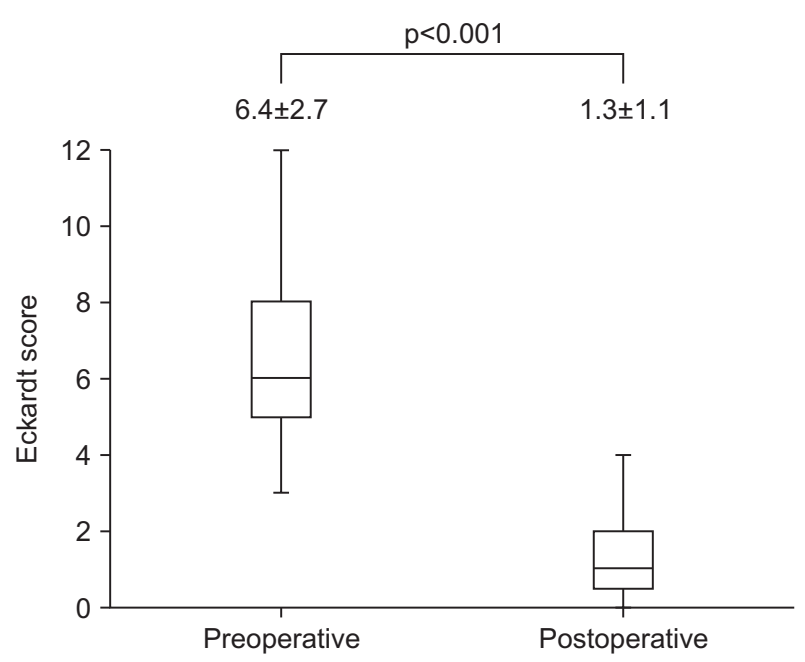

Fig. 2. Change in the Eckardt score after peroral endoscopic myotomy.

Table 2. Changes in Physiological Parameters before and after Peroral Endoscopic Myotomy

\begin{tabular}{|c|c|c|c|}
\hline Characteristic & Preoperative values & Postoperative values & p-value \\
\hline \multicolumn{4}{|l|}{ EndoFLIP } \\
\hline DI30mL, $\mathrm{mm}^{2} / \mathrm{mm} \mathrm{Hg}$ & $3.5 \pm 2.6$ & $11.4 \pm 6.0$ & $<0.001$ \\
\hline $\mathrm{DI} 40 \mathrm{~mL}, \mathrm{~mm}^{2} / \mathrm{mm} \mathrm{Hg}$ & $3.3 \pm 2.7$ & $12.5 \pm 7.4$ & $<0.001$ \\
\hline CSA30mL, $\mathrm{mm}^{2}$ & $71.7 \pm 67.1$ & $173.7 \pm 96.1$ & $<0.001$ \\
\hline CSA40mL, $\mathrm{mm}^{2}$ & $94.4 \pm 78.4$ & $263.6 \pm 124.8$ & $<0.001$ \\
\hline \multicolumn{4}{|l|}{$\mathrm{HRM}, \mathrm{mm} \mathrm{Hg}$} \\
\hline LES pressure & $30.3 \pm 18.1$ & $13.5 \pm 9.4$ & $<0.001$ \\
\hline IRP & $27.8 \pm 27.1$ & $11.4 \pm 6.8$ & $<0.001$ \\
\hline \multicolumn{4}{|l|}{ Questionnaire } \\
\hline Eckardt score & $6.4 \pm 2.7$ & $1.3 \pm 1.1$ & $<0.001$ \\
\hline Development of reflux symptoms & 9 & 37 & $<0.001$ \\
\hline \multicolumn{4}{|l|}{ Endoscopy } \\
\hline Reflux esophagitis & 3 & 24 & $<0.001$ \\
\hline LA:A & 1 & 7 & \\
\hline LA:B & 2 & 13 & \\
\hline LA:C & 0 & 4 & \\
\hline \multicolumn{4}{|l|}{ 24-hour $\mathrm{pH}$ monitoring } \\
\hline Incidence of GERD & 9 & 27 & $<0.001$ \\
\hline
\end{tabular}

Data are presented as mean \pm SD or number.

DI, distensibility Index; CSA, cross-sectional area; HRM, high-resolution manometry; LES, lower esophageal sphincter; IRP, integrated relaxation pressure; LA, Los Angeles; GERD, gastroesophageal reflux disease. 

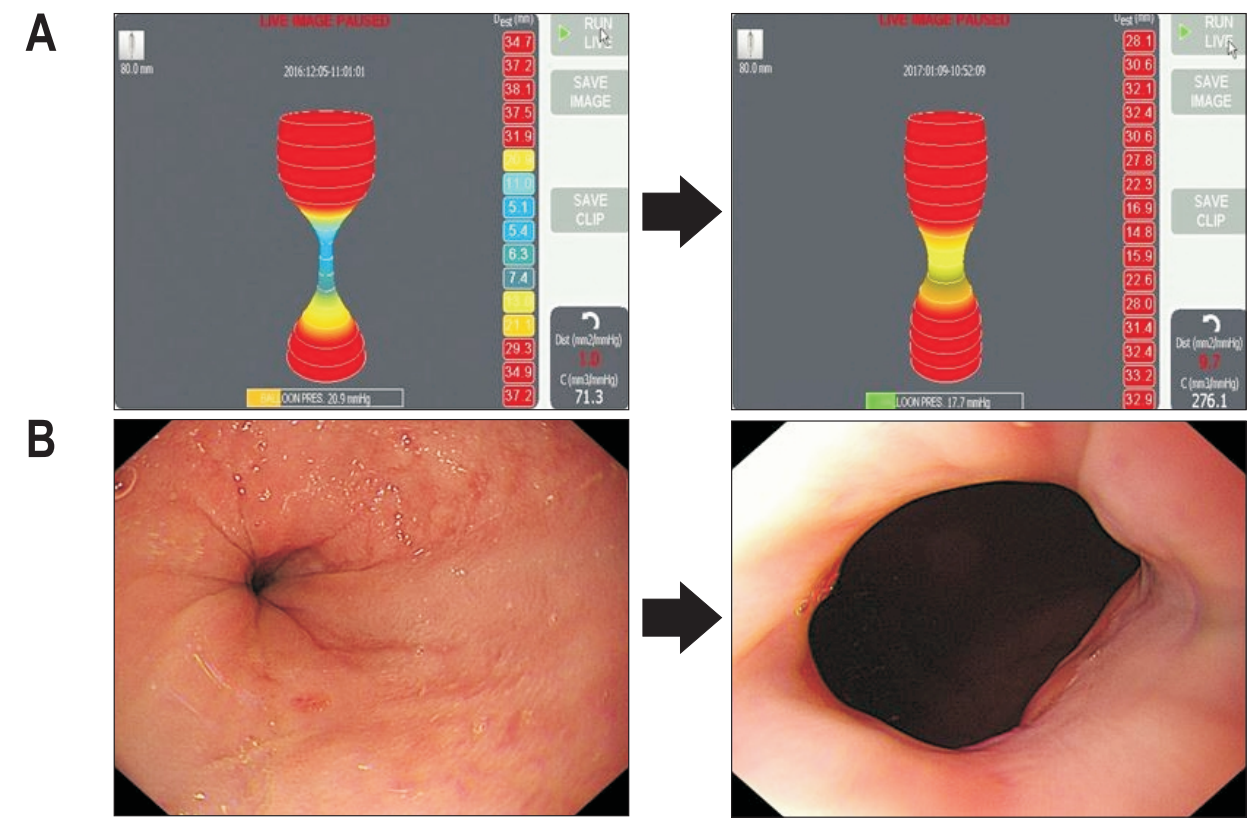

Fig. 3. Evaluation of the esophagogastric junction (EGJ) using distal esophageal spasm (EndoFLIP) and endoscopy before and after peroral endoscopic myotomy (POEM). (A) Examples of EndoFLIP displaying the EGJ with a $40-\mathrm{mL}$ volume inflation. Distensibility index (DI) before POEM is $1.0 \mathrm{~mm}^{2} / \mathrm{mm} \mathrm{Hg}$ (left) and DI after POEM is $9.7 \mathrm{~mm}^{2} / \mathrm{mm} \mathrm{Hg}$ (right). (B) Examples of endoscopy displayed the EGJ. The EGJ was tight before POEM (left) and The EGJ showing improvement in the opening diameter after POEM (right).

Table 3. Factors Associated with Incomplete Response to Peroral Endoscopic Myotomy

\begin{tabular}{|c|c|c|c|}
\hline & Postoperative ES $\geq 3$ ( $n=10)$ & Postoperative ES <3 (n=42) & p-value \\
\hline Male sex & $5(18.5)$ & $22(81.5)$ & 0.89 \\
\hline Age, yr & $44.5 \pm 17.6$ & $42.1 \pm 14.8$ & 0.65 \\
\hline BMI, $\mathrm{kg} / \mathrm{m}^{2}$ & $22.4 \pm 3.5$ & $22.4 \pm 3.8$ & 0.44 \\
\hline Follow-up duration, day & $209.9 \pm 121.0$ & $307.6 \pm 275.6$ & 0.58 \\
\hline Duration of disease, yr & $4.5 \pm 3.1$ & $6.0 \pm 8.6$ & 0.58 \\
\hline Maximal diameter of esophagus, $\mathrm{mm}$ & $30.5 \pm 9.1$ & $45.3 \pm 69.7$ & 0.53 \\
\hline Myotomy length, mm & $83.0 \pm 12.5$ & $92.9 \pm 17.9$ & 0.10 \\
\hline DI30mL, $\mathrm{mm}^{2} / \mathrm{mm} \mathrm{Hg}$ & $8.53 \pm 5.67$ & $14.00 \pm 8.36$ & 0.07 \\
\hline DI $40 \mathrm{~mL}, \mathrm{~mm}^{2} / \mathrm{mm} \mathrm{Hg}$ & $9.51 \pm 4.00$ & $14.17 \pm 8.16$ & 0.10 \\
\hline $\mathrm{CSA} 30 \mathrm{~mL}, \mathrm{~mm}^{2}$ & $167.00 \pm 86.01$ & $205.52 \pm 116.68$ & 0.35 \\
\hline CSA40mL mm ${ }^{2}$ & $256.67 \pm 80.66$ & $284.49 \pm 137.08$ & 0.56 \\
\hline Esophageal shape & & & 0.31 \\
\hline Sigmoid & 0 & 4 & \\
\hline Non-sigmoid & 10 & 38 & \\
\hline Full myotomy & 5 & 33 & 0.07 \\
\hline Selective myotomy & 5 & 9 & \\
\hline Post-DI30 or DI40 <7 & 6 & 5 & 0.001 \\
\hline Post-DI30 or DI $40 \geq 7$ & 4 & 37 & \\
\hline Decrease in LES pressure & 4 & 32 & 0.03 \\
\hline Increase in LES pressure & 6 & 10 & \\
\hline Decrease in IRP & 4 & 33 & 0.02 \\
\hline Increase in IRP & 6 & 9 & \\
\hline
\end{tabular}

Data are presented as number $(\%), m_{ \pm} a_{ \pm}$, or number.

ES, Eckardt score; BMI, body mass index; DI, distensibility index; CSA, cross-sectional area; LES, lower esophageal sphincter; IRP, integrated relaxation pressure.

\section{Incomplete response to POEM}

During the follow-up period, $10(19.2 \%)$ of the 52 patients had a final Eckardt score $>3$, with persistent symptoms of achalasia (Table 3). There was no significant difference in sex, age, body mass index (BMI), follow-up duration, duration of disease, 


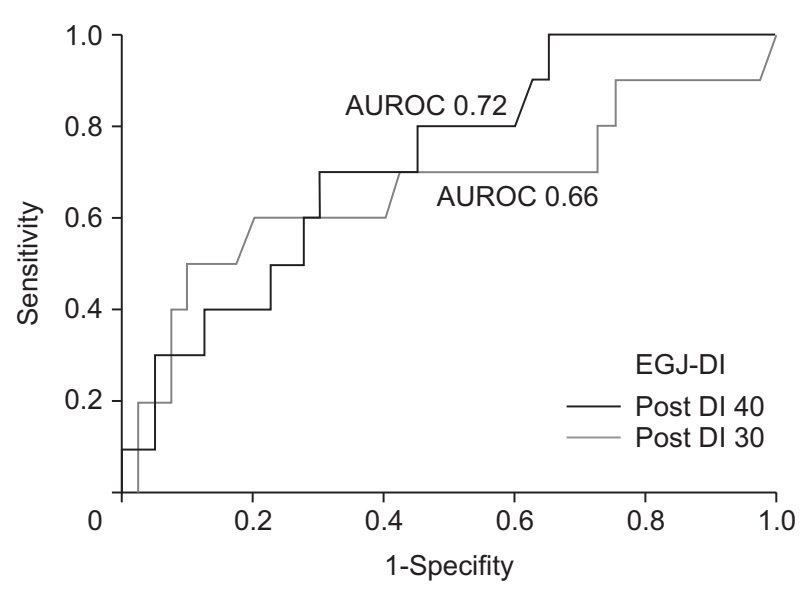

Fig. 4. Receiver operating characteristic (ROC) curve of esophagogastric junction (EGJ) distensibility index (DI) for incomplete response to peroral endoscopic myotomy.

AUROC, area under a ROC curve.

maximal esophageal diameter, and length of myotomy between the good response and incomplete response group. Although the patients with incomplete response showed relatively low DI30mL and DI40mL compared to the good response group during follow-up, this difference was not statistically significant ( $p>0.05$ ). However, a combination of DI30mL or DI40mL was correlated with incomplete response after POEM. Patients who exhibited postoperative DI30mL or DI40mL below seven showed a higher rate of incomplete response after POEM ( $p=0.001)$. The cutoff value of EGJ-DI was determined using ROC analysis (Fig. 4). The cutoff value for predicting incomplete response (Eckardt score $\geq 3$ ) after POEM was 6.6 at post-DI30mL (area under the ROC [AUROC], 0.66; 95\% confidence interval [CI], 0.44 to 0.88 ) and 9.9 at post-DI40mL (AUROC, 0.72; 95\% CI, 0.56 to 0.89). Therefore, we concluded that patients with a post-POEM DI (30 or $40 \mathrm{~mL}$ ) below seven had a significantly higher rate of incomplete response after POEM. On follow-up HRM, LES pressure increased paradoxically in 10 patients and IRP increased in nine patients. Paradoxical increase in LES pressure or IRP after POEM was also associated with incomplete response $(\mathrm{p}=0.023$ and $\mathrm{p}=0.012$, respectively). Type of achalasia, length of myotomy during POEM, pretreatment BMI, duration of disease, maximal diameter of esophagus, and achalasia on gastrografin esophagogram were not associated with incomplete response.

On multivariate analysis, an increase in IRP was associated with incomplete response ( $\mathrm{p}=0.047$ ) and low postoperative DI showed a stronger correlation with incomplete response $(\mathrm{p}=0.002)$. No variables except DI and IRP were significantly associated with incomplete response to POEM (Table 4).

\section{Complications}

Pneumoperitoneum (84 patients; 53\% of all patients) and subcutaneous emphysema (50 patients; $31 \%$ of all patients) were the most common POEM-related complications. There
Table 4. Multivariate Analysis for Prognostic Factors of Incomplete Response

\begin{tabular}{|c|c|c|}
\hline & \multicolumn{2}{|c|}{ Multivariate model } \\
\hline & OR $(95 \% \mathrm{CI})$ & p-value* \\
\hline Full thickness myotomy & $0.248(0.04-1.45)$ & 0.121 \\
\hline Postoperative DI30mL or DI40mL $<7$ & $14.10(2.29-86.82)$ & 0.004 \\
\hline Increase in LES pressure & $7.66(1.21-48.48)$ & 0.030 \\
\hline Increase in IRP & $7.30(1.22-43.62)$ & 0.029 \\
\hline
\end{tabular}

OR, odds ratio; CI, confidence interval; DI, distensibility index; LES, lower esophageal sphincter; IRP, integrated relaxation pressure. *Significant differences between groups were tested using binary logistic regression analysis.

were 15 patients with pneumomediastinum, 17 with pleural effusion, eight with atelectasis, six with pneumothorax (among which one had tension pneumothorax), four with pneumonia, and three with delayed esophageal hematoma. All patients had completely recovered with conservative treatment without surgical intervention.

\section{DISCUSSION}

POEM is a well-established minimal invasive treatment; it provides symptom relief equal to that provided by conventional laparoscopic Heller's myotomy, with less pain and a shorter recovery period. ${ }^{5}$ Prior studies have shown improvement in physiologic parameters of the EGJ after treatment in patients with achalasia. Hulselmans et al. ${ }^{10}$ demonstrated that lower posttreatment LES pressure is related to lower recurrence after pneumatic dilatation. Alderliesten et al. ${ }^{8}$ and Eckardt et al. ${ }^{7}$ reported that post-dilation LES pressure less than $10 \mathrm{~mm} \mathrm{Hg}$ is a significant predictive factor for a favorable long-term outcome. However, few studies have studied lower postoperative DI as a prognostic factor for patients with achalasia treated with POEM. In the present study, we evaluated the usefulness of EndoFLIP in assessing clinical outcomes of patients with achalasia after POEM. We hypothesized that DI has a predictive role during the followup period after POEM. EndoFLIP has been usually used intraoperatively for measurements of EGJ distensibility before and after POEM. However, due to the condition of operation room, it was difficult to measure EndoFLIP with all patients during procedure and sometimes EndoFLIP catheter could induce mucosal injury. Therefore, we perform EndoFLIP at outpatient clinic which can be easily applied in clinical settings.

As EndoFLIP is a real time examination for the EGJ, DI varies during measurement due to esophageal peristalsis. Therefore, it might be reasonable to perform EndoFLIP more than twice to evaluate the accurate physiology of the EGJ. In the present study, serial measurement of DI with 30-mL and 40-mL distension of the saline bag, for more than 1 minute each, was performed to validate the certainty of examination. We analyzed 
the correlation between incomplete response and low postoperative DI using a combination of DI30mL or DI40mL. Low postoperative DI $(<7)$ measured during volume-controlled distension with 30 or $40 \mathrm{~mL}$ of saline was associated with a high postoperative Eckardt score $(\geq 3)$, which suggests incomplete clinical response $(\mathrm{p}=0.002)$.

Unlike in prior studies, no significant correlation between LES pressure less than $10 \mathrm{~mm} \mathrm{Hg}$ and favorable outcome was seen in our study $(p=0.149)$. Because the mean postoperative LES pressure in our patient group was $13.6 \mathrm{~mm} \mathrm{Hg}$, relatively higher than $10 \mathrm{~mm} \mathrm{Hg}$, we could not obtain a conclusion consistent with that of the previous studies in terms of LES pressure. On the other hand, a paradoxical increase in LES pressure and IRP after POEM were noted in 10 and nine of 52 patients, respectively. Increase in LES pressure and IRP were also correlated to incomplete response. Those who exhibited increased IRP or LES pressure during follow-up had improved symptoms after POEM but complained of worsening symptoms over time. Sato et al. ${ }^{11}$ reported that muscle layer defect after POEM was replaced by fibrotic tissue in an animal model. Similarly, we hypothesize that fibrotic change in the submucosal tissues adjacent to the myotomy site resulted in impaired relaxation of the LES and thus elevated LES pressure and IRP. ${ }^{11}$

Patients with GERD are reported to exhibit high DI and patients with achalasia have low DI, when measured using EndoFLIP. Patients with GERD were reported to have elevated DI compared to healthy controls, particularly at 20- and 30$\mathrm{mL}$ volume distensions in a study by Kwiatek et al. ${ }^{12}$ In a study by Pandolfino et al., ${ }^{13}$ DI was lower in patients with untreated achalasia than in a healthy control group. However, there is a controversy regarding determination of normal values of DI in healthy controls. Rieder et al. ${ }^{14}$ reported that median DI30mL was 2.5 (range, 2.0 to 6.3) and DI40mL was 2.7 (range, 2.4 to 8.3) in four healthy volunteers. On the other hand, Rohof et al. ${ }^{4}$ reported that 15 healthy controls had a mean EGJ DI of $6.3 \pm 0.7$ with $50 \mathrm{~mL}$ volume distension, which was higher than that seen in patients with untreated achalasia. As the DI values that are considered normal in prior studies are different, it is reasonable to focus on the postoperative change in DI rather than the values themselves. In the present study, pretreatment DI30mL and DI40mL were $3.6 \pm 2.6$ and $3.3 \pm 2.8$ respectively, both slightly higher than the normal range presented in Rohof's study. ${ }^{4}$ Even though postoperative DI30mL and DI40mL were also slightly higher than those reported in prior studies, an increase in DI was noted in all patients after POEM. Almost all the patients enrolled in our study underwent full-thickness myotomy at the level of the LES; we believe that the extent of myotomy resulted in a higher postoperative DI.

Minimal pneumoperitoneum and subcutaneous emphysema were common adverse events after POEM. All instances of these two complaints were treated with fasting and short-term parenteral nutrition for 4 to 7 days; all the patients recovered com- pletely without surgical treatment. The development of GERD was also common after POEM. Forty-six percent of patients showed reflux esophagitis on endoscopy and 28\% of patients aggravated GERD on 24-hour pH monitoring after POEM. In these patients, by using ROC analysis, post-DI (30 or $40 \mathrm{~mL}$ ) over 10 was associated with GERD development. Almost all patients with GERD symptoms recovered with conservative treatment with proton pump inhibitors and mucoprotective agents. Two patients with intractable symptoms of GERD were treated with anti-reflux endoscopic surgery, which completely relieved their symptoms.

There are several limitations in our study. First, this study was based on retrospective data obtained from a single center. Second, as the mean follow-up duration was 288 days with a maximum follow-up duration of 4 years, we do not know how the postoperative Eckardt score changes over a period longer than 4 years. Finally, the number of patients enrolled in our study was too small to confirm our results. Even though 144 patients underwent POEM, only 52 among them had completed the evaluation needed for enrollment. Further studies with a larger number of patients are needed to investigate the associations between an increase in DI and incomplete outcome after POEM in patients with achalasia.

In conclusion, lower measurements of post-POEM DI were associated with an incomplete POEM response. However, higher post-POEM DI predisposed toward postoperative GERD. Based on our study, patients within a post-DI range (7 to $10 \mathrm{~mm}^{2}$ / $\mathrm{mm} \mathrm{Hg}$ ) had optimal symptomatic outcomes which can predict minimal dysphagia and minimal GERD after the procedure. Therefore, post-POEM DI at the EGJ using EndoFLIP is a useful index to predict the treatment response and postprocedure reflux of POEM in patients with achalasia.

\section{CONFLICTS OF INTEREST}

No potential conflict of interest relevant to this article was reported.

\section{REFERENCES}

1. Podas T, Eaden J, Mayberry M, Mayberry J. Achalasia: a critical review of epidemiological studies. Am J Gastroenterol 1998;93:2345-2347.

2. Triadafilopoulos G, Boeckxstaens GE, Gullo R, et al. The Kagoshima consensus on esophageal achalasia. Dis Esophagus 2012;25:337-348.

3. Boeckxstaens GE, Annese V, des Varannes SB, et al. Pneumatic dilation versus laparoscopic Heller's myotomy for idiopathic achalasia. N Engl J Med 201;364:1807-1816.

4. Rohof WO, Hirsch DP, Kessing BF, Boeckxstaens GE. Efficacy of treatment for patients with achalasia depends on the distensibility of the esophagogastric junction. Gastroenterology 2012;143:328- 
335.

5. Inoue H, Minami H, Kobayashi $\mathrm{Y}$, et al. Peroral endoscopic myotomy (POEM) for esophageal achalasia. Endoscopy 2010;42:265271.

6. Inoue $\mathrm{H}$, Sato $\mathrm{H}$, Ikeda $\mathrm{H}$, et al. Per-oral endoscopic myotomy: a series of 500 patients. J Am Coll Surg 2015;221:256-264.

7. Eckardt VF, Aignherr C, Bernhard G. Predictors of outcome in patients with achalasia treated by pneumatic dilation. Gastroenterology 1992;103:1732-1738.

8. Alderliesten J, Conchillo JM, Leeuwenburgh I, Steyerberg EW, Kuipers EJ. Predictors for outcome of failure of balloon dilatation in patients with achalasia. Gut 2011;60:10-16.

9. Armstrong D, Bennett JR, Blum AL, et al. The endoscopic assessment of esophagitis: a progress report on observer agreement. Gastroenterology 1996;111:85-92.

10. Hulselmans M, Vanuytsel T, Degreef T, et al. Long-term outcome of pneumatic dilation in the treatment of achalasia. Clin Gastroen- terol Hepatol 2010;8:30-35.

11. Sato H, Sagara S, Suzuki K, Terai S, Yahagi N. Assessments of histologic changes after peroral endoscopic myotomy. Gastrointest Endosc 2016;84:377-378.

12. Kwiatek MA, Pandolfino JE, Hirano I, Kahrilas PJ. Esophagogastric junction distensibility assessed with an endoscopic functional luminal imaging probe (EndoFLIP). Gastrointest Endosc 2010;72:272-278.

13. Pandolfino JE, de Ruigh A, Nicodème F, Xiao Y, Boris L, Kahrilas PJ. Distensibility of the esophagogastric junction assessed with the functional lumen imaging probe $\left(\mathrm{FLIP}^{\mathrm{TM}}\right)$ in achalasia patients. Neurogastroenterol Motil 2013;25:496-501.

14. Rieder E, Swanström LL, Perretta S, Lenglinger J, Riegler M, Dunst $\mathrm{CM}$. Intraoperative assessment of esophagogastric junction distensibility during per oral endoscopic myotomy (POEM) for esophageal motility disorders. Surg Endosc 2013;27:400-405. 